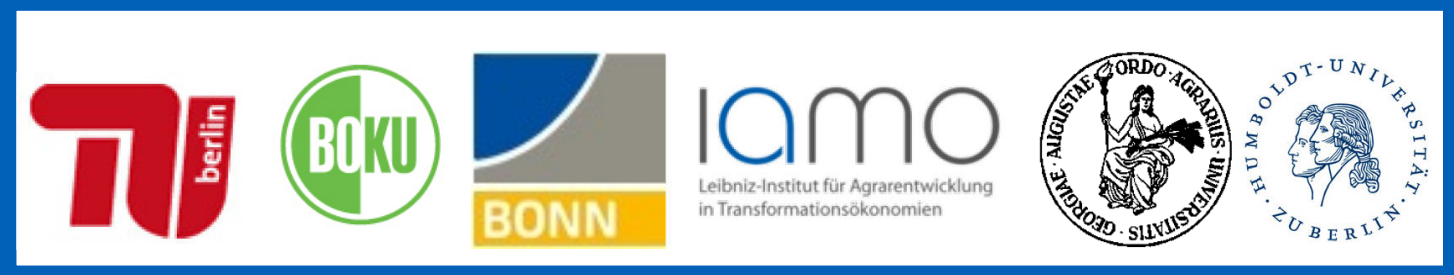

\title{
FORLAND
}

\section{Public preferences for pasture landscapes and the role of scale heterogeneity}

Henning Schaak, Oliver Musshoff

\section{FORLand-Working Paper 04 (2018)}

\section{Published by}

DFG Research Unit 2569 FORLand, Humboldt-Universität zu Berlin Unter den Linden 6, D-10099 Berlin

https://www.forland.hu-berlin.de (ㄷ) (1)

Tel +49 (30) 2093 46845, Email gabriele.wuerth@agrar.hu-berlin.de

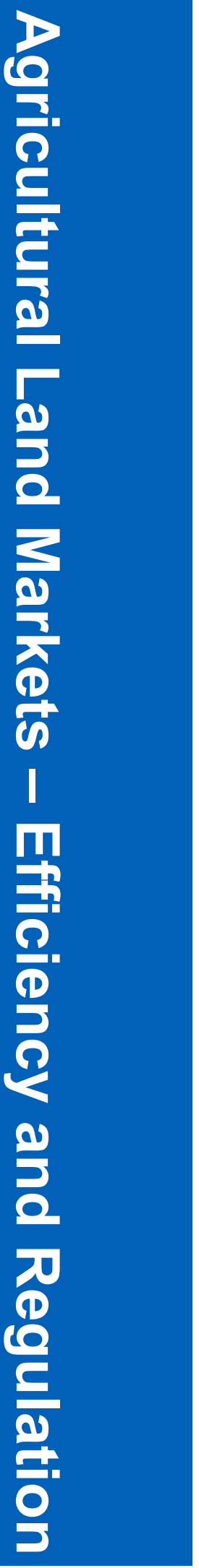




\title{
Public preferences for pasture landscapes and the role of scale heterogeneity
}

\author{
Henning Schaak *, Oliver Musshoff **
}

\section{October 2018}

\begin{abstract}
Despite its relevance for agricultural production, biodiversity and landscape aesthetics, grazing livestock is rarely considered in research on public landscape preferences. This paper studies public preferences for pasture usage in Germany by the means of a discrete choice experiment. The results indicate that there is a general willingness to pay (WTP) for livestock presence in landscape. The mean WTP is independent of its density. The paper discusses the implications of different econometric models and the role of preference heterogeneity on the results. The results show that a detailed analysis of the preference heterogeneity can provide deeper insights on their structure.
\end{abstract}

Keywords: Discrete-Choice-Experiment, Public Landscape Preferences, Livestock, Mixed Logit Model

JEL codes: $\quad$ C25, Q15

\section{Acknowledgements}

Henning Schaak gratefully acknowledges financial support from the Ministry for Science and Culture of Lower Saxony (MWK) through the collaborative research project "SAM, Analysis of Dairy Production: Grazing versus Indoor Housing of Dairy Cows", Support Code: ZN 2864. Oliver Musshoff gratefully acknowledges financial support from the German Research Foundation (DFG) through Research Unit 2569 "Agricultural Land Markets - Efficiency and Regulation".

* Henning Schaak, henning.schaak@agr.uni-goettingen.de

** Oliver Musshoff, Oliver.Musshoff@agr.uni-goettingen.de

Both authors are at University of Goettingen, Department for Agricultural Economics and Rural Development, Platz der Göttinger Sieben 5, 37073 Göttingen, Germany 


\section{Introduction}

In context of agriculture and environmental protection, changes in land use and landscapes are critical issues. They are also discussed in the context of related policies. One important topic is the development of pasture land and the way it is utilized by agriculture. Pastures take on numerous tasks and functions and contribute a great deal to preserve biodiversity. According to the German Federal Agency for Nature Conservation (BfN 2018), over half of the plant species in Germany have their habitat in pastures. Thus, the conservation of pastures is required for the preservation of the biodiversity level (Plachter and Hampicke 2010). Another important task of pastures is their role for climate protection. The conversion of pasture into arable land, as well as the production of peat, releases large amounts of CO2. Furthermore, most pastures bind $\mathrm{CO} 2$ for around 100 years (Poeplau et al. 2011). Many pastures in Germany are younger than 100 years, thus, they still have a positive impact on the reduction of CO2 (BfN 2014). Pastures also play a role in flood protection and erosion. Rivers and brooks are often surrounded by extensive pastures, which take over the function of floodplains and protect crops and villages (BfN 2014). In addition, an increasing rooting of the soil prevents soil erosion and keeps nutrients in the ground (Hampicke 2013).

While the pure decline of the pasture share has slowed down (primarily due to plow bans and related policy measures (BfN 2014), the focus of interest changed towards the specific usage form of the pasture. Here, primary attention is given to the usage form "grazing", which means that cattle (or other bovines) feed themselves on the pasture (cf. Blanchet, Moechnig and DeJong-Hughes 2000; Hodgson 1990). With respect to various aspects, it has been stressed that grazing can be a preferable usage form of pastures. It has been shown that pasture access can provide health and welfare benefits for dairy cows (Armbrecht et al. 2016; Keyserlingk et al. 2009). Under suitable conditions, grazing based milk production can also improve the economic performance of dairy production (Knaus 2016; Peyraud et al. 2010; Steinwidder et al. 2011; Thomet et al. 2011). This is closely linked with the exact embodiment of the grazing system. While extensive grazing systems are generally considered to be preferable with respect to biodiversity issues (Hampicke 2013), intensive grazing systems (e.g. rotational grazing) allow for a more efficient usage of the overgrowth (Hodgson 1990). Also, in the context of landscape aesthetics, grazing is important. Besides a small, mosaic structured appearance of agricultural plots, grazing cattle is a distinct feature of many traditional landscapes in Europe (Plachter and Hampicke 2010).

Despite these positive evaluations and the political relevance of the topic, little is known about public preferences towards the usage form of pastures (e.g. as hay meadow or for different grazing systems). Public landscape preferences are derived by letting citizens assess and valuate the aesthetic quality of landscapes (Rambonilaza and Dachary-Bernard 2007). A landscape can be defined as "the outdoor environment, natural or built, which can be directly perceived by a person visiting and using that environment" (Hull IV and Revell 1989). These preferences have been analyzed by numerous studies, reviews are e.g. given by Záková Kroupová et al. (2016) and van Zanten et al. (2014). Besides revealed preference methods like hedonic regression (Walls, Kousky and Chu 2015), the research in this field heavily relies on the usage of stated preference methods. Besides the contingent valuation method, an increasing share of studies utilize Discrete Choice Experiments (DCE) (Hoyos 2010). 
DCEs have the advantage that they allow for the derivation of the Willingness to Pay (WTP) for landscape changes (De Ayala Bilbao, Hoyos Ramos and Mariel Chladkova 2012). So far, research on public landscape preferences has rarely explicitly considered the presence of livestock in the landscape. In a meta-analysis of DCEs on landscape preferences, van Zanten et al. (2014) found that in only 14 out of 345 cases, studies used "presence of livestock" as an landscape attribute, while it was among the highest preferred landscape attributes. To the best of our knowledge, previous studies only differentiated between presence and absence of livestock and did not considered varying livestock densities. Still, if there are differences, this may be relevant for agricultural production, as different grazing systems require varying livestock densities. Related to the reported preference for visible livestock, research in consumer studies report higher preferences and a WTP for milk and beef products from a grazing based production (e.g. Ellis et al. 2009; Weinrich et al. 2014). It is worth noting that landscape evaluation studies typically focus on specific landscapes and often either survey inhabitants of, or tourists in a particular region of interest (van Zanten et al. 2014).

Taking previous results on consumer preferences for pasture milk products into account, the question arises, whether these preferences only have to be attributed to, e.g., perceived product quality differences or animal welfare benefits. The main objective of the paper is to study whether the idea of livestock presence itself also has a value, even when the consumer does not "use" the presence (speaking in terms of use and non-use values of the total economic value concept (Millennium Ecosystem Assessment 2003), as existence or heritage values may be present. In order to better understand this issue, we divert from the common approach of considering a specific landscape; instead, we construct a DCE within a general scenario, which does not consider a particular region. It further focusses on the visibility of livestock and its density, rather than the related agricultural products. Other attributes concern the landscapes structure. We use graphical representations of the choice sets and use a cost attribute to estimate consumers' WTP for the livestock presence and the structural elements. For the DCE, a representative sample of consumers from all over Germany was surveyed. The experimental design and the survey process were chosen in order to identify the WTP for typical elements of pasture landscapes in Germany.

Although preferable in the context of our study, the application of DCEs can lead to issues when the observed preferences are not homogenous. In order to account for potential preference heterogeneity, multiple approaches have been developed. The Mixed Multinomial Logit model (MIXL) (McFadden and Train 2000; Train 2009) allows for varying parameters, which are expressed by a continuous heterogeneity distribution and has been widely applied in the literature ${ }^{1}$. Alternative approaches capture the preference heterogeneity by allowing different latent classes (Boxall and Adamowicz 2002), or so-called "scale-heterogeneity" (Fiebig et al. 2010). Fiebig et al. (2010) introduced a generalized model (Generalized Multinomial model, GMNL), which allows for preference and scale heterogeneity.

1 While the MIXL allows the parameters to be correlated, the term is commonly referred to a specification with uncorrelated parameters (Fiebig et al. 2010; Hess and Train 2017). The present paper follows this notation. 
So far, best to the authors' knowledge there are currently no applications of the GMNL in the field of landscape evaluations. This assessment was also given by De Ayala Bilbao et al. (2012), who conclude that "there seems to be a need for analyzing the behavior of this model in this kind of applications". Given the different regional origins of the participants in our study, it is reasonable to assume that the participant's preferences are not homogenous. In order to study whether preference and/or scale heterogeneity is present in the context of landscape evaluations, we compare the results of the GMNL with the well-established MIXL. To further exploit the effects of scale heterogeneity on the preference structure, we also compare the distributions of the conditional means of individual parameters (Fiebig et al. 2010; Sarrias and Daziano 2017).

Through the approach presented above, the paper contributes to the literature in several ways. By explicitly considering the livestock density on the pasture, the paper is the first to go beyond the simple presence or absence of cattle in the landscape. Furthermore, it is the first to explicitly consider the preferences on the national scale. Lastly, the role of scale heterogeneity and the behavior of the GMNL, in the context of landscape preferences, has not been explicitly addressed before now.

The remainder of this paper is structured as follows: In the second section, we describe the DCE. In Section 3, first the survey design and data collection are presented. Then, the collected data is described and econometric models used for their analysis are outlined. The results are presented and discussed in section 4. The paper ends with conclusions (section 5).

\section{The experiment}

As discussed in the previous section, this paper builds on the method of DCE. In recent years, DCE's have gained increasing attention, especially in the context of environmental evaluations (Hoyos 2010). In a DCE, different sets of choice situations are used, in which the participant has to choose the alternative which he or she prefers. The choice situations are described by a number of attributes, for which the levels are varied. DCEs are based on the Random Utility theory (McFadden 1974). Assuming that the utility is derived from the attributes of the good (cf. Lancaster's theory of value; Lancaster 1966), and by the introduction of a stochastic component, it allows the analysis of the stated choices under utility maximization. In order to calculate the WTP for individual attributes and levels, DCEs regularly include a cost attribute. In the following subsections, we first motivate and describe the scenario, attributes, and levels of the DCE and then describe the graphical representations of the choice sets.

\subsection{Scenario, attributes and levels}

Given the scope of this paper, a suitable DCE was designed. For this, attributes and their respective levels, as well as a scenario, had to be developed. Considering prior research on landscape preferences and taking own deliberations into account, suitable attributes and their respective levels have been chosen. They are presented in Table 1. The attribute presence of livestock describes the presence and number of dairy livestock on the pasture. The different levels differentiate between no livestock, and a low, medium, or high number of livestock. Structuredness of the pasture is an attribute which is represented by a parceling of the grazing area. This is done by fences which divide the pasture into additional land parcels. 
The different levels distinguish between no additional parcels, or a low, medium or high number of additional parcels on the main plot. Another attribute are the point landscape elements, including trees and individual bushes. These elements are classified as either present or not present. The attribute linear landscape elements describe hedges and larger groups of bushes. As for the point landscape elements, these elements are either present or not present. In order to include the monetary dimension, a cost attribute was included. The cost per household per year can take the values $0 €, 15 €, 30 €, 45 €, 60 €, 75 €$, and $90 €$.

\section{Table 1: Attributes and levels of the Discrete Choice Experiment}

\begin{tabular}{ll}
\hline Attribute & Level \\
\hline Presence of livestock (No. of cattle) & None, low, medium, high \\
Structuredness of the pasture (No. of land parcels) & None, low, medium, high \\
Point landscape elements & Not present/ Present \\
Linear landscape elements & Not present/ Present \\
Cost per household per year & $0 €, 15 €, 30 €, 45 €, 60 €, 75 €, 90 €$ \\
\hline
\end{tabular}

As the focus of this paper is on general preferences for the landscape attributes, a general scenario was chosen. In the scenario, it is outlined that societal development will lead to more homogenous landscapes with less structural elements. Additionally, the share of grazing cattle will decrease towards a very small share. Under these assumptions, it is reasonable that the typical landscape in the future will look like presented in the left of Figure 1. In order to slow down or even reverse this development, a new pasture protection program is to be designed. The participant now has to choose between multiple program possibilities, which are designed to lead to other expected landscape structures. These programs are associated with additional costs for consumers, which are the sum of additional taxes, fees, and higher product prices, etc. The participants are informed that they will be confronted with several sets of two pictures representing the possible expected outcomes of such a policy. They are then asked to select the alternative to which they prefer. As participants often state an exaggerated WTP in hypothetical decision situations, the scenario description includes a cheap talk-script, which explicitly addresses this issue (Carlsson, Frykblom and Lagerkvist 2005).

Figure1: left side: Basic landscape with all attributes at their lowest level; right side: Landscape with all attributes at their highest level

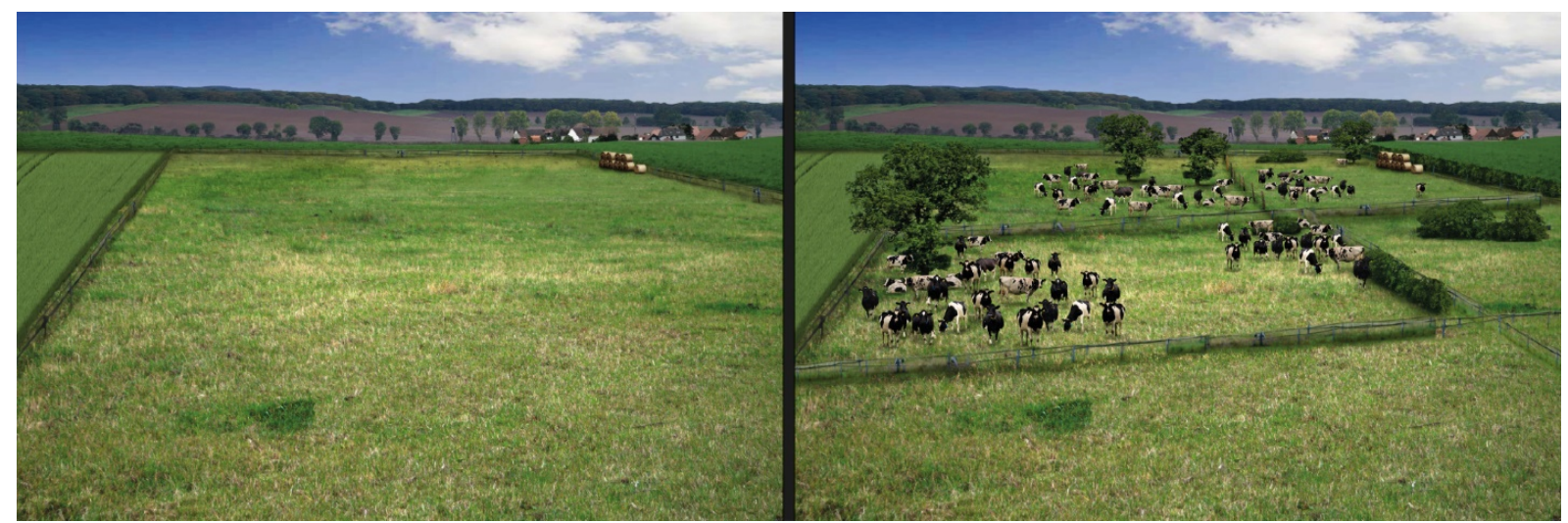

Source: Authors' illustration 


\subsection{Graphical representation}

In the present study, the attributes of the DCE, with the exception of the cost attribute, are graphically represented. The basis is an artificially created picture of a landscape (van Zanten et al. 2016) (shown in Figure 1). Using different photos taken near Hildesheim, middle of Germany, the basis picture was generated. In order to avoid potential biases due to other landscape elements (e.g. mountains) or regional particularities, the landscape was constructed in an unspecific way. The image shows a landscape in June, and is dominated by a large pasture in the fore- and middle ground with some cultivated cropland on the sides. The pasture size is approximately 10 hectares, excluding any livestock, trees or bushes. In the right-hand corner of the background-image, a small village represents the rural character of this region. Furthermore, some trees and a forest are visible within the frame. According to the final experimental designs, the various attribute levels are gradually added, whereby the basic conditions, such as light, weather conditions and/or the perspective, remain the same. The right side of Figure 1 illustrates the landscape with all attributes at their highest level. The different images were created with Adobe Photoshop CS6.

\section{Material and Methods}

\subsection{Survey design and data collection}

Based on the selected attributes and levels, a DCE was designed. It is an unlabeled DCE, including two alternatives and an opt-out-alternative, which corresponds to an alternative with all attributes at the lowest level. The design of the experiment followed a sequential process. In a first small pilot study, the participants were asked to state their maximum WTP for several possible choice alternatives which were presented. These statements were used to determine realistic levels for the cost attribute. Next, an efficient design with uninformative priors (Rose and Bliemer 2009) was created. This design was the basis for a second pilot study. Based on its results, informative priors were obtained and used for the determination of a Bayesian-Defficient design (Rose and Bliemer 2009). The final DCE consisted of 12 choice sets.

The DCE is part of an online survey. In the first part of the survey, the DCE was carried out. Following the DCE, a questionnaire had to be answered. The survey started with a welcome address and the motivation of the survey. Then, the participants were introduced to the scenario of the DCE and confronted with an example choice set. After a multiple-choice question, controlling for the participants understanding of the experiment's introduction, the DCE followed. Participants who answer the control question incorrectly twice were excluded from the survey. Following the DCE, the participants were presented with questions regarding their perception of the environment, including questions on how important the different attributes were for their decisions and if any of them were ignored. Participants who choose the opt-out-option at least one time were asked for their motivation.

During the second part of the survey, information concerning the participants attitudes related to the studies issues, such as the participant's attitude towards environmental or animal welfare issues was gathered. Relying on previous research on preferences for cultural landscapes (e.g. Scarpa et al. 2011), the study asks for the individual landscape usage behavior, personal connection towards agriculture, milk, and beef production and related information, such as the 
membership in environmental protection groups. Finally, information about socio-economic variables, such as age, gender, and income were gathered. On the closing page, the participants were thanked for their participation and contact information was provided.

\subsection{Sample description}

The data collection was conducted by an online-sampling company in September and October of 2017. The sample contained participants from Germany. By enforcing quotas, it was ensured that the participants are representative regarding the age, household income, federal state of residence and size of the place of residence for the German population (based on information from the German federal statistical office; (Destatis (Statistisches Bundesamt) 2017b; Destatis (Statistisches Bundesamt) 2017a). In total, 475 participants completed the survey, with 449 participants being included in the study sample. The descriptive statistics of the sociodemographic characteristics is presented in Table 2.

Table 2: Descriptive statistics

\begin{tabular}{|c|c|c|}
\hline & Mean & SD \\
\hline Age (in years) & 45.47 & 14.60 \\
\hline Gender $(0=$ male, $1=$ female $)$ & $46.55 \%$ & \\
\hline Marital status (not married $=0$, married $=1$ ) & $44.95 \%$ & \\
\hline Household size & 2.45 & 1.21 \\
\hline \multicolumn{3}{|l|}{ Household income } \\
\hline$<1300 €$ & $9.58 \%$ & \\
\hline$<1700 €$ & $8.24 \%$ & \\
\hline$<2600 €$ & $23.16 \%$ & \\
\hline$<3600 €$ & $18.26 \%$ & \\
\hline$<5000 €$ & $24.05 \%$ & \\
\hline$>5000 €$ & $16.07 \%$ & \\
\hline Personal relationship towards agriculture $(0=$ no, $1=$ yes $)$ & $35.41 \%$ & \\
\hline Farmer $(0=$ no, $1=$ yes $)$ & $0.44 \%$ & \\
\hline \multicolumn{3}{|l|}{ Landscape type around the place of residence } \\
\hline Coast landscapes & $5.12 \%$ & \\
\hline Forest landscapes and forest dominated landscapes & $27.62 \%$ & \\
\hline Richly structured cultural landscapes & $13.14 \%$ & \\
\hline Open cultural landscapes & $22.72 \%$ & \\
\hline Mining landscapes & $1.11 \%$ & \\
\hline Urban agglomeration & $30.29 \%$ & \\
\hline
\end{tabular}

Notes: $(\mathrm{N}=449)$,

Source: Authors' calculation

The participants are on average 45.5 years of age. This is below the overall German mean, but corresponds with the mean of the group of the 18-69 year old, the age span which was offered by the sampling company. Nearly half of the participants were female, $45 \%$ of the participants being married. As previously mentioned, the household income is representative of the German population. The average household size is 2.5 , ranging from 1 to 9 persons. Although $35.4 \%$ of the participants stated a personal relationship towards agriculture (such as growing up on a farm, or having farming relatives), only 2 participants were actual farmers. The majority of the participants identified their local surrounding as either an area of urban 
agglomeration or a forest (or forest dominated) landscape (applying the categorization of Gharadjedaghi et al. 2004).

\subsection{Methodology}

As highlighted in the introduction, the experimental data were analyzed by the means of the MIXL and the GMNL. In this section, we only briefly discuss their properties and refer to the main references ${ }^{2}$. The MIXL is a generalization of the Multinomial Logit model, which allows for individual preference parameters (McFadden and Train 2000; Train 2009). The person $i$ 's random utility for alternative $j$ and choice situation $t$ is given by

$$
U_{i j t}=\boldsymbol{x}_{i j t}^{\mathrm{T}} \boldsymbol{\beta}_{i}+\epsilon_{i j t}
$$

with $i=1, \ldots, N ; j=1, \ldots, J$ and $t=1, \ldots, T_{i}$. The observed alternative attributes are contained in the vector $\boldsymbol{x}_{i j t}^{\mathrm{T}}$ with the dimension $K \times 1$. $\epsilon_{i j t}$ is the i.i.d. extreme value type 1 idiosyncratic error term. The parameter vector $\boldsymbol{\beta}_{i}$ is unobserved and assumed to vary in the population. $A$ common assumption is that $\boldsymbol{\beta}_{i}$ follows a multivariate normal distribution. Under this assumption, $\boldsymbol{\beta}_{i}$ can be written as

$$
\boldsymbol{\beta}_{i}=\boldsymbol{\beta}+\mathbf{L} \boldsymbol{\eta}_{i}
$$

Here $\boldsymbol{\beta}$ is the mean vector and $\mathbf{L}$ the lower-triangular Cholesky factor of the covariance-matrix of the distribution of $\boldsymbol{\beta}_{i}$ and $\boldsymbol{\eta}_{\boldsymbol{i}}$ follows $N(\mathbf{0}, \boldsymbol{I})$.

The MIXL is widely applied in the literature (Keane and Wasi 2012). In order to allow for scale heterogeneity, the GMNL, proposed by Fiebig et al. (2010), generalizes the MIXL, where

$$
\boldsymbol{\beta}_{\boldsymbol{i}}=\sigma_{i} \boldsymbol{\beta}+\left[\gamma+\sigma_{i}(1-\gamma)\right] \mathbf{L} \boldsymbol{\eta}_{\boldsymbol{i}} .
$$

Here, $\sigma_{i}$ is a scale factor of the idiosyncratic error term, and varies among individuals. It is assumed to be log normal distributed with the mean $\bar{\sigma}$ and the standard deviation ${ }^{3} \tau$. Besides the mean vector $\boldsymbol{\beta}$, the variance of residual taste heterogeneity $\mathbf{L} \boldsymbol{\eta}_{\boldsymbol{i}}$ also varies with the scale. The extent is controlled by the scalar $\gamma \cdot \gamma$ can take any value, and can lead to different interpretations of the model structure (Keane and Wasi 2012).

Fiebig et al. (2010) point out two special cases. First, when $\gamma=1$, the model reduces to $\boldsymbol{\beta}_{\boldsymbol{i}}=$ $\sigma_{i} \boldsymbol{\beta}+\mathbf{L} \boldsymbol{\eta}_{\boldsymbol{i}}$. In this case, referred to as GMNL-I, the residual taste heterogeneity is independent of the scaling factor of $\boldsymbol{\beta}$. In the second case, referred to as GMNL-II, $\gamma=0$, therefore the residual scale heterogeneity is proportional to $\sigma_{i}$, as the model reduces to $\boldsymbol{\beta}_{\boldsymbol{i}}=\sigma_{i}\left(\boldsymbol{\beta}+\mathbf{L} \boldsymbol{\eta}_{\boldsymbol{i}}\right)$. The interpretation of the two models is straightforward. In case of the GMNL-I, the mean parameters are scaled by an individual, random factor. In case of the GMNL-II, both the mean parameters and the taste heterogeneity are scaled. The GMNL allows to account for "extreme", "almost lexicographic" as well as more "random" preferences (Fiebig et al. 2010).

2 The used notation follows Sarrias and Daziano (2017)

3 Given by $\sigma_{i}=\exp \left(\bar{\sigma}+\tau v_{i}\right)$ with $v_{i} \sim N(0,1)$. Note, that $\bar{\sigma}$ has to be normalized, see Fiebig et al. (2010) for details. 
Still, it has been argued that incautious interpretations of such models can lead to incorrect conclusions about the preference structure of the respondents (Davis, Burton and Kragt 2016). Related, Hess and Train (2017) emphasize significant results of a GMNL that are not a definite proof of scale heterogeneity, as the scale parameter may also capture other sources of heterogeneity.

Noteworthy, we estimated all models in the WTP-space (Sonnier, Ainslie and Otter 2007), meaning that we directly estimated WTP for the attribute instead of ordinary preference parameters. For both models, it is possible to derive conditional estimates for each individual (Sarrias and Daziano 2017; Train 2009). We calculated the conditional expected mean for each individual in the sample and derived the posterior distribution of the estimated means. For the technical details, we refer to Sarrias and Daziano (2017).

\section{Results and Discussion}

The estimation results for the choice decisions are presented in Table 3. All levels were included as dummy variables. Estimations were completed using the 'gmnl'-package (Sarrias and Daziano 2017) for the software ' $R$ ' (R Core Team 2016). The models were estimated in WTP-space requiring a fixed cost-coefficient of -1 , thus, the estimated mean parameters could be directly interpreted in $€$ for each respective level.

Comparing the results of the MIXL and the GMNL in terms of AIC and BIC indicated that the GMNL ought to be preferred. As one can see, the estimated $\gamma$ is close to 1 , which showed that the model goes towards the special case GMNL-I. Re-estimating the model with $\gamma$ restricted at 1 (thus, explicitly estimating the GMNL-I) led to an even smaller AIC and BIC. The result is presented in the third column ${ }^{4}$. As both models fit the data better, we therefore have to conclude that the GMNL-I as well as the GMNL outperform the more common MIXL in our study. Additionally to the better model fit, the significant estimate for $\mathrm{T}$ indicates that scale heterogeneity is present, both in the GMNL and the GMNL-I. Comparing the GMNL and the GMNL-I, information criteria, as well as a likelihood ratio-test, indicated that the GMNL-I is to be preferred. When comparing the estimates of the MIXL and the GMNL variants, one can see that the estimates for the mean parameters were roughly the same magnitude, while most of the estimated standard deviations distinctly changed. For the mean parameters for "Structuredness: low" and "Structuredness: medium" we observed a sign change, although only the negative values in the MIXL are statistically significantly different from zero.

\footnotetext{
${ }^{4}$ For completeness, we also estimated a GMNL-II model. With respect to information criteria, it falls behind all other estimated models and is therefore not presented.
} 
Table 3: Regression results in WTP-space

\begin{tabular}{|c|c|c|c|c|c|c|}
\hline & \multicolumn{2}{|c|}{ MIXL } & \multicolumn{2}{|c|}{ GMNL } & \multicolumn{2}{|c|}{ GMNL-I } \\
\hline Mean Parameter & Est. & SE & Est. & SE & Est. & SE \\
\hline Livestock: low & $81.24^{* * *}$ & (2.63) & $87.10^{* \star *}$ & (3.01) & $87.12^{* \star *}$ & (2.97) \\
\hline Livestock: medium & $85.78^{* * *}$ & (3.90) & $90.95^{* * *}$ & $(2.20)$ & $90.98^{* * *}$ & (2.19) \\
\hline Livestock: high & $82.89^{* * *}$ & (2.75) & $81.58^{* * *}$ & $(4.78)$ & $81.20^{\star * *}$ & $(4.71)$ \\
\hline Structuredness: low & $-5.67^{*}$ & (2.74) & 6.34 & $(4.24)$ & 6.48 & $(4.25)$ \\
\hline $\begin{array}{l}\text { Structuredness: } \\
\text { medium }\end{array}$ & $-4.44^{*}$ & (1.99) & 7.63 & $(4.24)$ & 7.31 & $(4.02)$ \\
\hline Structuredness: high & $6.08^{* *}$ & (2.31) & 7.52 & $(2.52)$ & $7.55^{\star *}$ & $(2.52)$ \\
\hline Point Elements & $79.83^{* * *}$ & $(2.16)$ & $73.72^{\star \star}$ & $(2.40)$ & $73.73^{* * *}$ & (2.42) \\
\hline Linear Elements & $6.02^{* * *}$ & (1.63) & $8.28^{* * *}$ & $(1.83)$ & $8.24^{* *}$ & $(1.76)$ \\
\hline Cost & $-1.00^{a}$ & & $-1.00^{a}$ & & $-1.00^{a}$ & \\
\hline \multicolumn{7}{|l|}{ SD parameter } \\
\hline Livestock: low & $64.63^{* * *}$ & (3.03) & $31.60^{* \star \star}$ & (5.39) & $30.99^{* * *}$ & (5.33) \\
\hline Livestock: medium & $106.75^{* * *}$ & (5.28) & $35.99^{* * *}$ & (9.08) & $36.43^{* * *}$ & (8.99) \\
\hline Livestock: high & $119.12^{* * *}$ & (4.93) & $92.70^{* * *}$ & $(14.78)$ & $92.94^{* * *}$ & (14.57) \\
\hline Structuredness: low & $33.82^{* * *}$ & (4.93) & $37.89^{* * *}$ & $(7.35)$ & $38.26^{* * *}$ & $(7.54)$ \\
\hline $\begin{array}{l}\text { Structuredness: } \\
\text { medium }\end{array}$ & $12.41^{* * *}$ & (2.81) & $28.41^{* * *}$ & $(7.25)$ & $29.70^{* * *}$ & (7.11) \\
\hline Structuredness: high & $36.59^{* * \star}$ & $(2.18)$ & $24.14^{* \star *}$ & (6.63) & $25.22^{* * *}$ & (6.72) \\
\hline Point Elements & $17.49^{* * *}$ & (3.59) & $25.50^{* * *}$ & (5.48) & $25.19^{* * *}$ & (5.64) \\
\hline Linear Elements & $17.95^{\star \star \star}$ & $(2.05)$ & 0.62 & $(5.19)$ & 2.35 & $(5.07)$ \\
\hline \multicolumn{7}{|l|}{ Global parameter } \\
\hline T & & & $1.041^{* * *}$ & $(0.126)$ & $1.031^{* * *}$ & $(0.114)$ \\
\hline $\mathrm{Y}$ & & & $1.002^{* * *}$ & $(0.160)$ & $1.000^{\mathrm{a}}$ & \\
\hline \multicolumn{7}{|l|}{ Model statistics } \\
\hline $\mathrm{N}$ & \multicolumn{2}{|c|}{5,833} & \multicolumn{2}{|c|}{5,833} & \multicolumn{2}{|c|}{5,833} \\
\hline log likelihood & \multicolumn{2}{|c|}{$-4,559.67$} & \multicolumn{2}{|c|}{$-4,555.10$} & \multicolumn{2}{|c|}{$-4,554.05$} \\
\hline $\mathrm{AIC}$ & \multicolumn{2}{|c|}{$9,151.34$} & \multicolumn{2}{|c|}{$9,146.21$} & \multicolumn{2}{|c|}{$9,142.09$} \\
\hline $\mathrm{BIC}$ & \multicolumn{2}{|c|}{$9,256.81$} & \multicolumn{2}{|c|}{$9,264.86$} & \multicolumn{2}{|c|}{$9,254.15$} \\
\hline
\end{tabular}

Notes: using 1,000 halton draws, panel structure of the data was taken into account; ${ }^{*} p<0.05,{ }^{* *} p<0.01, p<0.001$; ${ }^{a}$ : fixed parameter

Source: Authors calculations

For a first interpretation of the results, we consider the GMNL-I. As one can see, the estimated mean parameters for the presence of livestock are significantly different from zero and range from 81-91 $€$ per household per year. Nevertheless, comparing the estimated parameters using multiple comparisons (using the Bonferroni correction) revealed that they do not differ significantly from each other (all adjusted $p$-values $>0.17$ ). This indicated that the average participant has a significant WTP for livestock presence in the landscape, although it is independent of the number of animals. The estimated standard deviations of the livestock parameters only significantly differ between the low, respectively medium and the high level (with adjusted $\mathrm{p}$-values $<0.001$ ).

This means that the preference heterogeneity was greater for the high level. For the "Structuredness" mean parameters, we found positive, but small estimates (compared to the livestock parameters), of which only the one for the "high" number of land parcels is significant 
( $8 €$ per household per year). Yet, a multiple comparison revealed that the estimates themselves are not significantly different (all adjusted p-values close to 1).

The same holds for the estimated standard deviations (all adjusted p-values $>0.59$ ). The cautious interpretation of these results leads to the conclusion, that the participants have no clear preferences for the structuredness of the pasture.

For the presence of point and linear landscape elements, we found significantly positive estimates. However, the estimate for point landscape elements is almost nine times as large as the one for linear elements, and significantly different with $p<0.001$ ( $74 €$ vs. $8 €$ ). This leads to the conclusion that consumers generally prefer the presence of structural elements, but the preference is higher for elements like trees. Furthermore, only the estimated standard deviation for the point landscape elements is significant, thus, the preference for the linear landscape elements is homogenous among the participants. These results indicate that there is a general preference and WTP of German consumers for cultural landscapes with present livestock, regardless of the individual place of residence. These general results are in line with previous findings in the literature (cf. van Zanten et al. 2014).

Apart from the estimates for structuredness of the landscape and the statistically significant of scale parameter, the results of the MIXL and the two GMNL variants appear qualitatively comparable. As previously discussed, it has been argued that researchers have to appropriately address the scale heterogeneity in order to avoid improper conclusions. The basic implication of the significant estimate for $\tau$ indicates that the scale parameter $\sigma_{i}$ significantly varies among the individuals in our sample. With respect to the GMNL-I, this implies that the mean parameter vector $\boldsymbol{\beta}$ is either up- or downscaled for each individual. This indicates that the interpretation of the estimates presented above may have to be modified. As a result, the conditional means of the individual parameters were also calculated.

The distributions of the estimates for all attributes in both models are presented in Figures 24. In all figures, the upper row shows the distributions for the MIXL, while the lower row shows the distributions of the GMNL-I. According to Fiebig et al. (2010), these distributions can also be interpreted as the posterior distributions of the mean parameters. Figure 2 presents the estimates for the livestock levels, Figure 3 the estimates for the structuredness of the landscape, while Figure 4 for the linear and point landscape elements.

Two particularities can be observed in all figures. First, the distributions for the MIXL are more symmetric than the distributions from the GMNL-I. Here, most distributions are, to some degree, left-skewed. Secondly, most MIXL-distributions have more local peaks and, in case of the point and linear landscape elements, can even be described as bimodal distributions.

Both aspects are particularly distinct for the livestock presence (Figure 2). In case of the medium level of livestock presence, the median value for the WTP-distribution is over $10 €$ higher as the mean (101.33 € against $90.80 €$ ). The distribution of the high livestock presence level also reveals that some individuals have a negative WTP for the level, which applies for $5 \%$ of the individuals. This is an interesting result, as it implies that these individuals would prefer a landscape without livestock presence over one with the highest level of present livestock. 
Given that the share of negative WTPs is around $1 \%$ for the other two levels, this could indicate that some individuals consider the livestock density being too high. This could lead to issues when bringing agricultural practice and the societal preference together, as intensive rotational grazing systems require high animal densities on a particular plot.

Figure 2: Distributions of the conditional WTP estimates for the Livestock dummies

MIXL
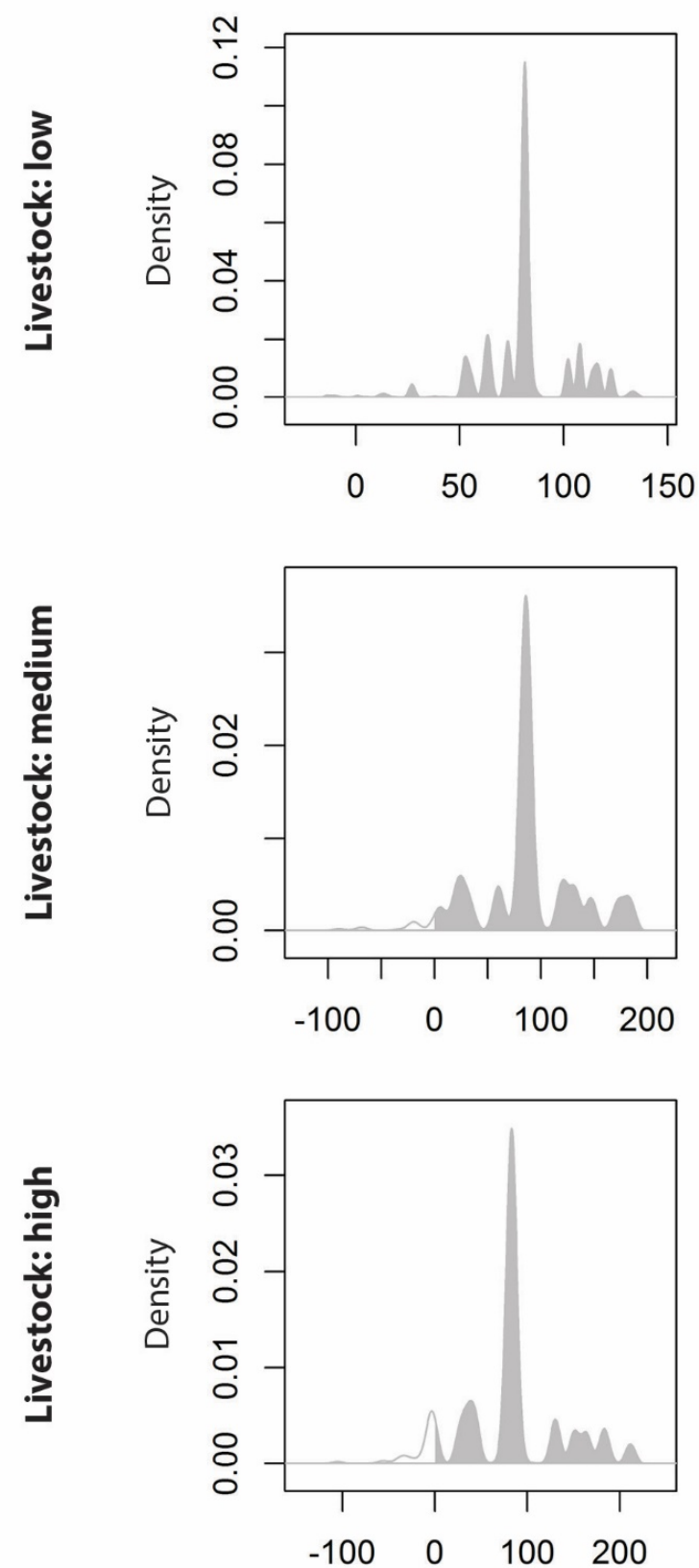

GMNL-I
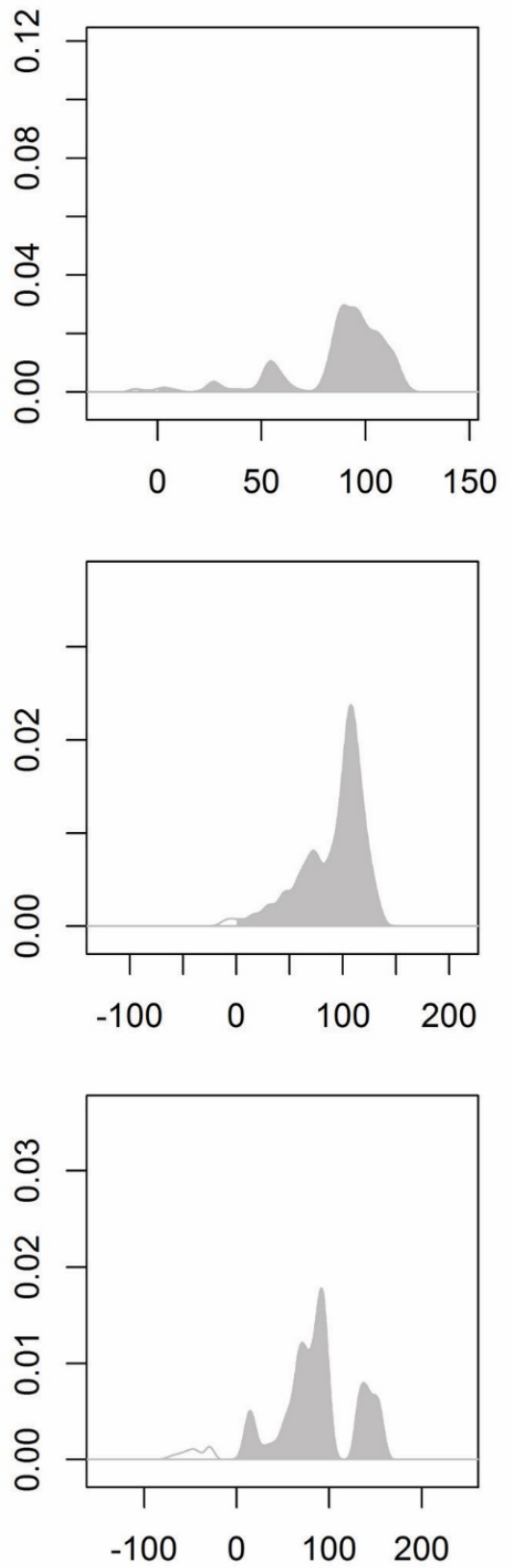

Note: the grey area gives the proportion with a positive WTP Source: Authors' illustration 
The distributions presented in Figure 3 also show the general features discussed before, but less pronounced. The differences in the share of negative WTPs have to be attributed to the differences in the estimated mean parameters of the initial models.

Figure 3: Distributions of the conditional WTP estimates for the Structuredness dummies

MIXL
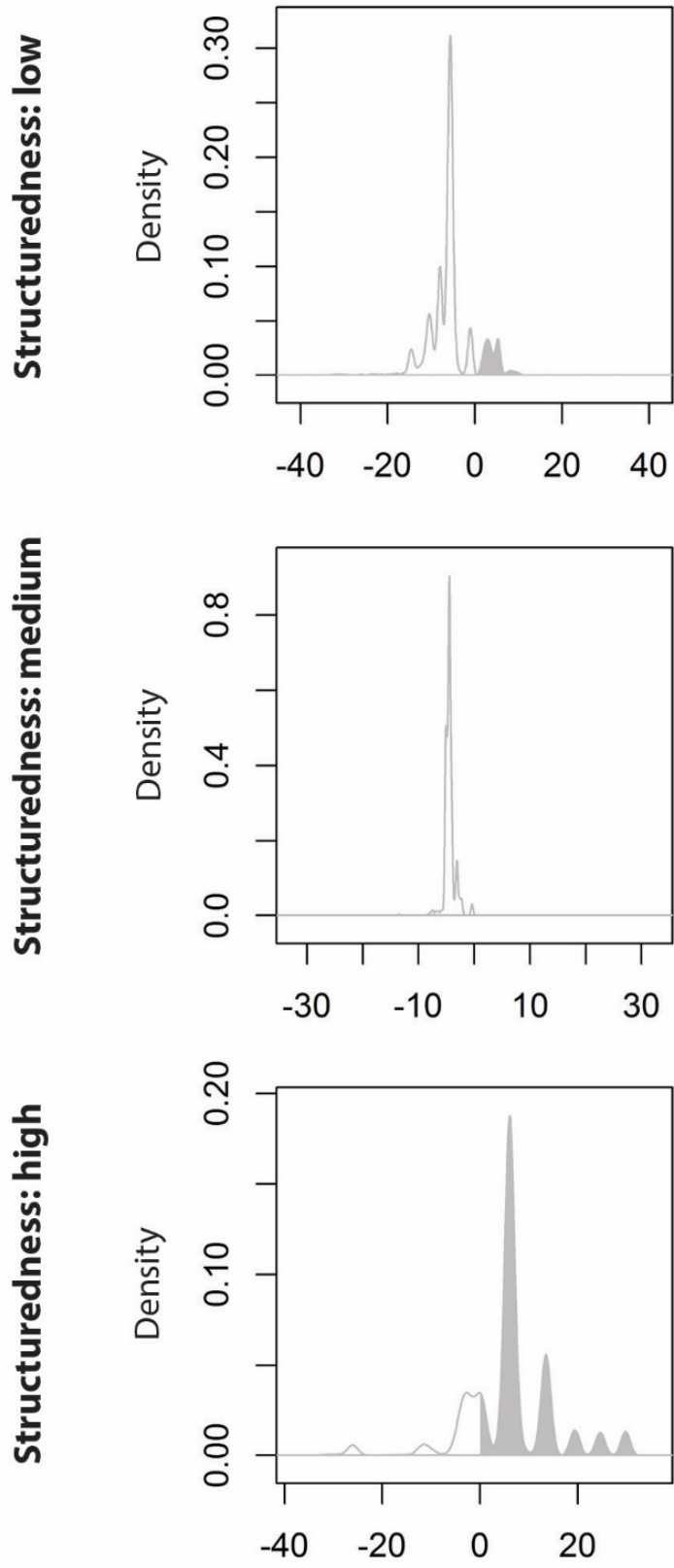

GMNL-I
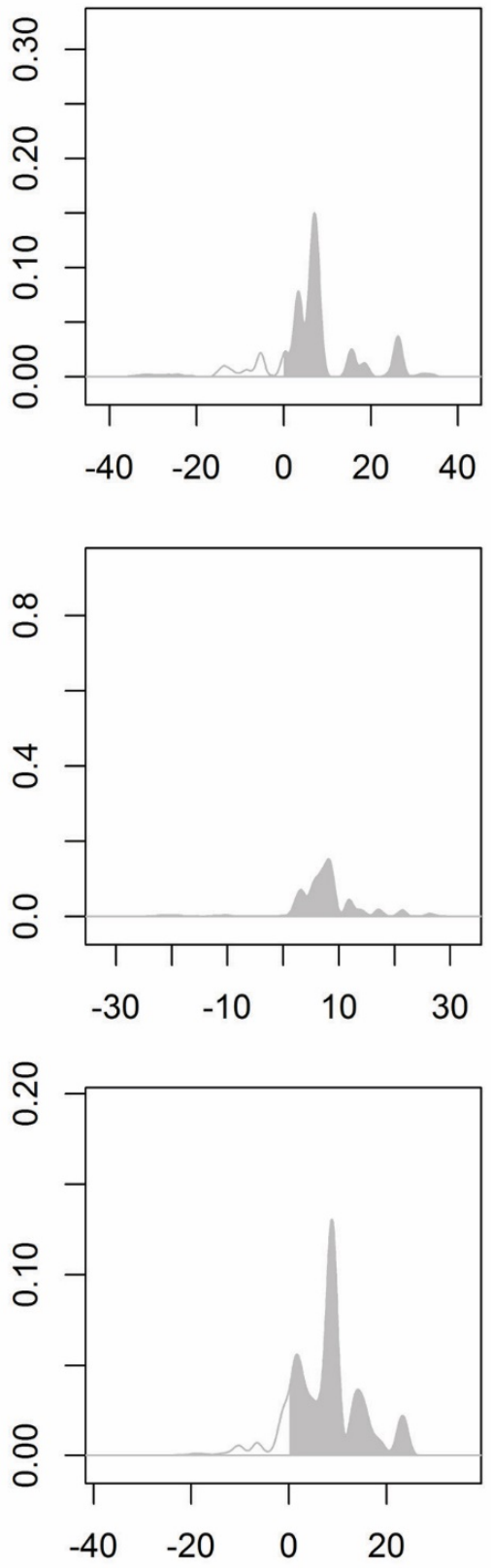

Note: the grey area gives the proportion with a positive WTP Source: Authors' illustration 
Figure 4: Distributions of the conditional WTP estimates for the Landscape elements dummies

MIXL
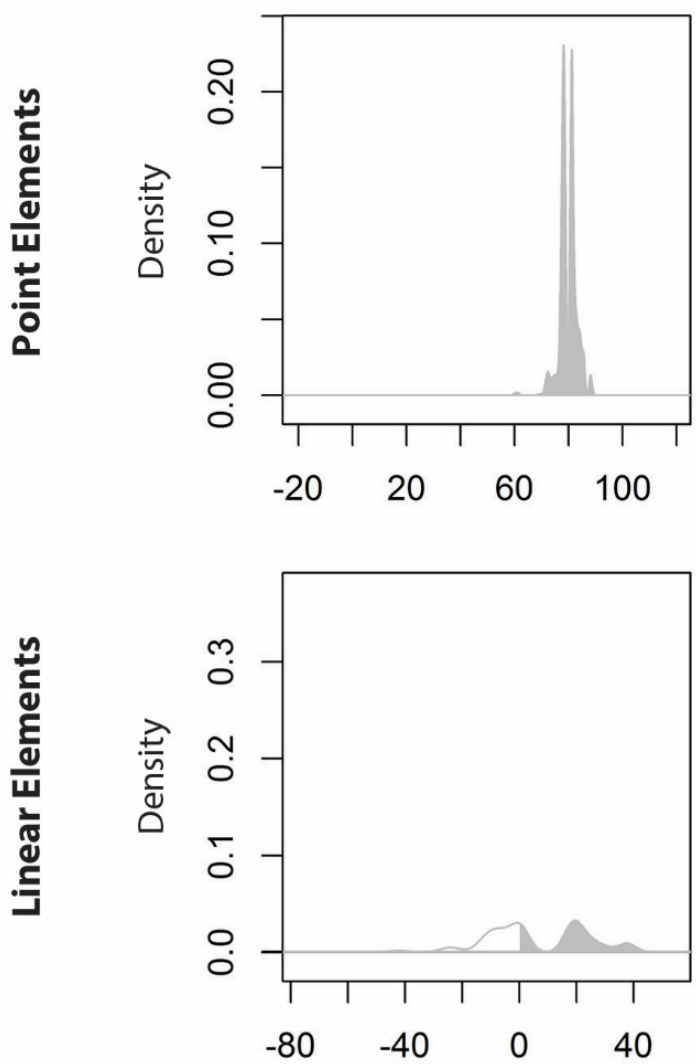

GMNL-I
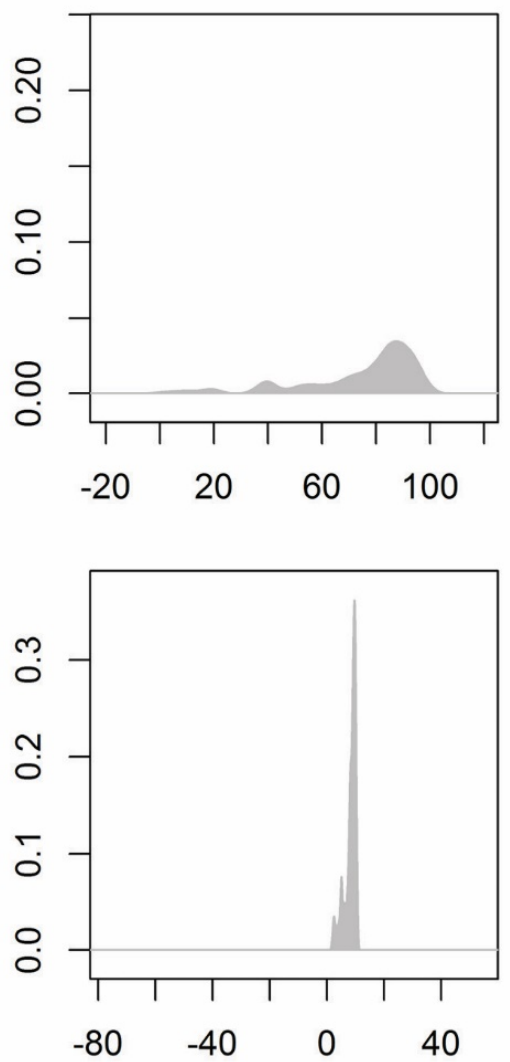

Note: the grey area gives the proportion with a positive WTP Source: Authors' illustration

In Figure 4, the densities of for GMNL-I are also distinctly left-skewed. Also, the densities of the MIXL feature two clear peaks. If the focus of the paper would be on the MIXL, this would also lead to interesting implications. The peaks of the linear elements are at values with different signs, and a very low density for the estimated mean parameter. This makes statements about the mean value of the population problematic, especially when being the basis for a policy recommendation. The plot for linear elements in the GMNL-I also illustrate another aspect of the GMNL. Although the estimated standard deviation is not significantly different from zero, the individual mean still follows a distribution. This results from the randomness of $\sigma_{i}$, and illustrates that the role of the two sources of heterogeneity can be accounted by the model.

\section{Conclusions}

Given the role of pastures for the agricultural production, the environmental protection and the corresponding societal expectations, this study aimed to improve the understanding of public preferences towards cultural pasture landscapes in Germany. The focus was on the role of livestock presence in these landscapes on the country level. 
In order to analyze the public WTP for pasture landscapes, a discrete choice experiment was conducted. Further, the paper sought to provide insights whether the application of the GMNL could be important in context of landscape preference evaluations.

The results showed that there is a general preference and WTP for livestock presence in the landscape and that its mean does not depend on the observed livestock density. Based on the utilized representative sample, which consists of consumers from all over Germany and rural as well as urban areas, it becomes evident that this general result holds for the German public as a whole. The results further showed that the usage of the GMNL can be appropriate in the context of landscape preference evaluations, as it improved the estimates fit to the data. Taking individual parameters into account provided further insights into the distribution of WTPs in the population. There are some consumers, who have strong preferences against high livestock densities and even reveal negative WTPs for the high level of livestock presence. Nevertheless, the key result, that a strong public preference for livestock presence on the country level, remains, strengthening the overall evidence in the literature.

The results presented in this study can be used to support environmental and agricultural policy planning in Germany, where grazing-based production and pastures are viable topics in regional agricultural politics. While the pasture based dairy production has been mainly discussed in the context of marketing actions recently, the question arises whether pasture access should be explicitly addressed in the broader agricultural policy actions, especially given the size of the estimated average WTP of over $80 €$ per household per year.

Although the study helps to extend the understanding of public landscape preferences in Germany, open research questions remain. Most importantly, the study currently focuses on the preference heterogeneity and does not take sociodemographic characteristics as explanatory variables into account. Further, the study does not use the individuals' residence to identify possible regional differences. Suitable approaches require cautious analysis and increase the computational complexity considerably.

The presented results have implications for possible further research. First, future studies in the field of landscape evaluation should consider whether models like the GMNL are a suitable alternative to currently established models. Secondly, given the usage of such a model, the request of the theoretical literature for an explicit assessment of the estimated scale heterogeneity also applies in context landscape evaluations. Although scale heterogeneity may appear as a technical detail at first, the study has shown that addressing its implications is required when the GMNL is applied. This can potentially help to give policy recommendations, which better reflect different public groups with varying preferences. Generally, when applying models that allow for individual heterogeneity, researchers should consider individual parameters' distribution rather than only the estimated means, as the posterior mean distribution is not necessarily a normal distribution, and the estimated mean parameters therefore should not be interpreted under the assumption of a normal distribution. Thirdly, regarding the limitations of the present study, future research should incorporate sociodemographic characteristics and consider the individuals' residence in order to identify possible sources of the observed preference heterogeneity. 


\section{Literature}

Armbrecht, L., C. Lambertz, D. Albers, and M. Gauly. 2016. "Does access to pasture affect welfare in dairy cows?" In 67th Annual Meeting of the EAAP, 29.08.-01.09.2016. Belfast.

Blanchet, K., H. Moechnig, and J. DeJong-Hughes. 2000. Grazing systems planning guide. University of Minnesota, Extension Service.

Boxall, P.C., and W.L. Adamowicz. 2002. "Understanding Heterogeneous Preferences in Random Utility Models: A Latent Class Approach.” Environmental and Resource Economics 23(4):421-446.

Bundesamt für Naturschutz (BfN). 2018. "Grassland conservation in Germany." Grassland conservation in Germany. https://www.bfn.de/en/activities/agriculture/grasslandconservation-in-germany.html [Accessed May 23, 2018].

Bundesamt für Naturschutz (BfN) ed. 2014. Grünland-Report - Alles im grünen Bereich? Bonn.

Carlsson, F., P. Frykblom, and C.J. Lagerkvist. 2005. "Using cheap talk as a test of validity in choice experiments." Economics Letters 89(2):147-152.

Davis, K.J., M. Burton, and M. Kragt. 2016. "Discrete choice models: scale heterogeneity and why it matters." No. 235373, University of Western Australia, School of Agricultural and Resource Economics. https://EconPapers.repec.org/RePEc:ags:uwauwp:235373.

De Ayala Bilbao, A., D. Hoyos Ramos, and P. Mariel Chladkova. 2012. "Landscape valuation through discrete choice experiments: Current practice and future research reflections."

Destatis (Statistisches Bundesamt). 2017a. "Bevölkerung und Erwerbstätigkeit." https://www.destatis.de/DE/Publikationen/Thematisch/Bevoelkerung/Bevoelkerungsstan d/Bevoelkerungsfortschreibung2010130157004.pdf [Accessed July 6, 2017].

Destatis (Statistisches Bundesamt). 2017b. "Einkommen, Einnahmen und Ausgaben privater Haushalte 2015."

https://www.destatis.de/DE/Publikationen/Thematisch/EinkommenKonsumLebensbedin gungen/EinkommenVerbrauch/EinnahmenAusgabenprivaterHaushalte2150100157004. pdf [Accessed July 6, 2017].

Ellis, K., K. Billington, B. McNeil, and D. McKeegan. 2009. "Public opinion on UK milk marketing and dairy cow welfare." Animal Welfare 18(3):267-282.

Fiebig, D.G., M.P. Keane, J. Louviere, and N. Wasi. 2010. "The Generalized Multinomial Logit Model: Accounting for Scale and Coefficient Heterogeneity." Marketing Science 29(3):393-421.

Gharadjedaghi, B., R. Heimann, K. Lenz, C. Martin, V. Pieper, A. Schulz, A. Vahabzadeh, P. Finck, and U. Riecken. 2004. "Verbreitung und Gefährdung schutzwürdiger Landschaften in Deutschland." Natur und Landschaft 79(2):71-81.

Hampicke, U. 2013. Kulturlandschaft und Naturschutz: Probleme - Konzepte - Ökonomie. Wiesbaden: Springer Spektrum.

Hess, S., and K. Train. 2017. "Correlation and scale in mixed logit models." Journal of Choice Modelling 23:1-8.

Hodgson, J.G. 1990. Grazing management: science into practice. Harlow: Longman Scientific \& Technical.

Hoyos, D. 2010. "The state of the art of environmental valuation with discrete choice experiments." Ecological Economics 69(8):1595-1603.

Hull IV, R.B., and G.R.B. Revell. 1989. "Issues in sampling landscapes for visual quality assessments." Landscape and Urban Planning 17(4):323-330.

Keane, M., and N. Wasi. 2012. "Comparing Alternative Models of Heterogeneity in Consumer Choice Behavior: Models of Heterogeneity in Consumer Choice Behavior." Journal of Applied Econometrics 28:1018-1045.

Keyserlingk, M.A.G. von, J. Rushen, A.M. de Passillé, and D.M. Weary. 2009. "Invited review: The welfare of dairy cattle-Key concepts and the role of science." Journal of Dairy Science 92(9):4101-4111.

Knaus, W. 2016. "Perspectives on pasture versus indoor feeding of dairy cows." Journal of the Science of Food and Agriculture 96(1):9-17. 
Lancaster, K.J. 1966. "A New Approach to Consumer Theory." Journal of Political Economy 74(2):132-157.

McFadden, D. 1974. "Conditional logit analysis of qualitative choice behavior." In P. Zarembka, ed. Frontiers in Econometrics. New York: Academic Press, pp. 105-142.

McFadden, D., and K. Train. 2000. "Mixed MNL Models for Discrete Response." Journal of Applied Econometrics 15(5):447-470.

Millennium Ecosystem Assessment ed. 2003. Ecosystems and human well-being: a framework for assessment. Washington, DC [u.a.]: Island.

Peyraud, J., A. Van den Pol-Van Dasselaar, P. Dillon, and L. Delaby. 2010. "Producing milk from grazing to reconcile economic and environmental performances." Grassland Science in Europe 15:865-879.

Plachter, H., and U. Hampicke eds. 2010. Large-scale Livestock Grazing. Berlin, Heidelberg: Springer Berlin Heidelberg. http://link.springer.com/10.1007/978-3-540-68667-5 [Accessed August 1, 2016].

Poeplau, C., A. Don, L. Vesterdal, J. Leifeld, B. Van Wesemael, J. Schumacher, and A. Gensior. 2011. "Temporal dynamics of soil organic carbon after land-use change in the temperate zone - carbon response functions as a model approach: SOIL ORGANIC CARBON AND LAND-USE CHANGE." Global Change Biology 17(7):2415-2427.

R Core Team. 2016. R: A Language and Environment for Statistical Computing. Vienna, Austria: R Foundation for Statistical Computing. https://www.R-project.org.

Rambonilaza, M., and J. Dachary-Bernard. 2007. "Land-use planning and public preferences: What can we learn from choice experiment method?" Landscape and Urban Planning 83(4):318-326.

Rose, J.M., and M.C.J. Bliemer. 2009. "Constructing Efficient Stated Choice Experimental Designs." Transport Reviews 29(5):587-617.

Sarrias, M., and R. Daziano. 2017. "Multinomial Logit Models with Continuous and Discrete Individual Heterogeneity in R: The gmnl Package." Journal of Statistical Software 79(2). http://www.jstatsoft.org/v79/i02/ [Accessed January 11, 2018].

Scarpa, R., S. Notaro, J. Louviere, and R. Raffaelli. 2011. "Exploring Scale Effects of Best/Worst Rank Ordered Choice Data to Estimate Benefits of Tourism in Alpine Grazing Commons." American Journal of Agricultural Economics 93(3):813-828.

Sonnier, G., A. Ainslie, and T. Otter. 2007. "Heterogeneity distributions of willingness-to-pay in choice models." Quantitative Marketing and Economics 5(3):313-331.

Steinwidder, A., W. Starz, L. Podstatzky, J. Gasteiner, R. Pfister, H. Rohrer, and M. Gallnböck. 2011. "Einfluss des Abkalbezeitpunktes von Milchkühen auf Produktionsparameter bei Vollweidehaltung im Berggebiet." Züchtungskunde 83:203215.

Thomet, P., E. Cutullic, W. Bisig, C. Wuest, M. Elsaesser, S. Steinberger, and A. Steinwidder. 2011. "Merits of full grazing systems as a sustainable and efficient milk production strategy." Grassland Science in Europe 16:273-285.

Train, K.E. 2009. Discrete choice methods with simulation. Cambridge university press.

Walls, M., C. Kousky, and Z. Chu. 2015. "Is What You See What You Get? The Value of Natural Landscape Views." Land Economics 91(1):1-19.

Weinrich, R., S. Kühl, A. Zühlsdorf, A. Spiller, and others. 2014. "Consumer attitudes in Germany towards different dairy housing systems and their implications for the marketing of pasture raised milk." International Food and Agribusiness Management Review 17(4):205-222.

Záková Kroupová, Z., M. Havlíková, P. Hálová, and M. Malý. 2016. "Economic Valuation of Mountain Landscapes and Ecosystems: A Meta-Analysis of Case Studies." Agris on-line Papers in Economics and Informatics VIII(3):103-112.

van Zanten, B.T., P.H. Verburg, M.J. Koetse, and P.J.H. van Beukering. 2014. "Preferences for European agrarian landscapes: A meta-analysis of case studies." Landscape and Urban Planning 132:89-101.

van Zanten, B.T., I. Zasada, M.J. Koetse, F. Ungaro, K. Häfner, and P.H. Verburg. 2016. "A comparative approach to assess the contribution of landscape features to aesthetic and recreational values in agricultural landscapes." Ecosystem Services 17:87-98. 